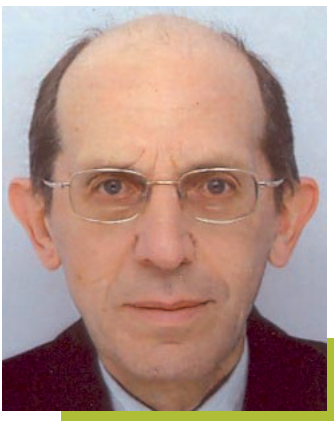

\title{
Réforme de la fiscalité directe locale - attention à la taxe foncière sur les propriétés bâties due par les entreprises
}

\author{
Mots-clés: fiscalité locale - taxe foncière sur les propriétés bâties - imposition \\ des entreprises - impôts de production
}

Le poids de l'imposition des entreprises à la taxe foncière sur les propriétés bâties est sous-estimé et risque de s'amplifier après la suppression de l'essentiel de la taxe d'habitation. Cette augmentation d'un impôt de production pénaliserait les entreprises, notamment les entreprises petites et moyennes implantées hors des métropoles.

'actualité des finances publiques locales est actuellement accaparée par la réforme de _ la fiscalité directe revenant aux collectivités locales, plus particulièrement par les vifs débats tournant autour des modalités de compensation par l'État de la suppression progressive de la taxe d'habitation frappant les résidences principales.

L'orientation et le contenu de ces débats marquent ainsi un intérêt plus particulier sur le devenir des ressources propres tirées d'une taxe appelée à disparaître, plutôt que sur le devenir du seul impôt direct d'un montant significatif dont l'évolution continuera à dépendre du pouvoir de taux des exécutifs communaux, à savoir la taxe foncière sur les propriétés bâties.

Ce devenir mériterait pourtant un intérêt au moins aussi important compte tenu des enjeux qu'il représente, non seulement pour les particu- 
liers propriétaires de leur logement, mais aussi pour les entreprises propriétaires des bâtiments et des équipements frappés par cette taxe.

En effet, le risque de surimposition paraît aussi préoccupant pour les entreprises que pour les ménages, bien que, s'agissant des entreprises, il ne soit quasiment jamais, ni évoqué, ni mesuré. Pourtant, ce risque mériterait d'être pris en compte au regard des mesures à prendre pour sauvegarder leur compétitivité et leur pérennité.

\section{La taxe foncière sur les propriétés bâties due par les entreprises au sein de la fiscalité directe locale n'est pas appréciée à sa juste valeur}

\section{A. La progression de la pression fis- cale exercée sur les ménages et les entreprises au titre de la taxe fon- cière sur les propriétés bâties est plus importante que pour la taxe d'habi- tation}

Au cours des trois dernières années, de 2016 à 2018, le produit de la taxe foncière sur les propriétés bâties (TFPB) a progressé globalement de $10,5 \%$ pour l'ensemble des collectivités qui la percevaient, communes, établissements publics intercommunaux à fiscalité propre et départements.

Cette progression est due, à la fois, à l'augmentation des bases d'imposition (" effet base ») de $5,8 \%$ et à l'augmentation des taux d'imposition (" effet taux) de 4,4\%. Elle a été plus rapide que pour la taxe d'habitation (TH) dont le produit, au cours de la même période, n'a progressé que de $4,5 \%$, dont $2,6 \%$ dus à «l'effet base » et 1,8\% dus à «l'effet taux».

Sur la période passée récente, on relève ainsi, au-delà de "l'effet base », une progression au niveau national du taux moyen d'imposition de TFPB supérieure de 2,6 points à celui de $\mathrm{TH}$.

Cette surimposition risque de s'accroître encore davantage avec la disparition totale programmée de la TH d'ici 2023, s'agissant de la TH imposée sur les logements constituant la résidence principale des particuliers et du fait du transfert aux communes, à compter de 2021, de la part départementale de la TFPB.

\section{B. Un risque de surimposition mal ap- précié pour les entreprises}

Les analyses portant sur la fiscalité directe locale ne réservent, en général, aucune place particulière à la TFPB due spécifiquement par les entreprises. Dans ces analyses, au contraire, l'évolution de la TFPB est appréciée globalement sous la rubrique trompeuse de "taxes sur les ménages » qui regroupe, à la fois, celle pesant sur les ménages et celle pesant sur les entreprises, ce qui exclut cette dernière de la rubrique « taxes sur les entreprises » censée pourtant regrouper l'ensemble de la fiscalité directe locale due par les entreprises.

Ce type de classification ne conduit pas à porter un regard particulier sur la place spécifique prise par la TFPB due par les entreprises au sein de la fiscalité directe locale. Pourtant, le risque de surimposition pèse aussi bien sur les ménages que sur les entreprises du fait de la disparition progressive de la plus grande part de la $\mathrm{TH}$ et du transfert aux communes de la part départementale de la TFPB, mais la part due par les entreprises $n^{\prime}$ est jamais mise en exergue malgré le poids significatif qu'elle représente dans l'ensemble du produit rapporté par la TFPB.

En effet, en 2018, la TFPB due par les entreprises s'est élevée à 12,2 Md€́1, soit 36,3\% des 33,6 Md€ rapportés par la TFPB dans son ensemble.

Pour être apprécié globalement, le risque de surimposition des entreprises au niveau local doit aussi prendre en compte les autres impôts directs recouvrés sur les entreprises au profit des collectivités locales. En 2018, les impôts les plus importants à ce titre se sont élevés au total à 25,7 Md€, dont $8 \mathrm{Md}$ pour la cotisation foncière d'entreprise (CFE) pour laquelle l'assiette relève de la même valeur locative que celle des biens passibles de la TFPB, et 17,72 Md€ pour la cotisation sur la valeur ajoutée des entreprises (CVAE).

Au total et en prenant en compte la TFPB, c'est donc un produit de près de $38 \mathrm{Md}$ qui a été perçu par les collectivités locales en 2018 auprès des entreprises au titre des trois impôts précités.

Selon l'INSEE, au lendemain du remplacement, en 2011, de la taxe professionnelle par la cotisation économique territoriale, le produit des trois impôts s'était élevé à $26 \mathrm{Md} €$, dont 5,2 Md€ pour la CFE, 11,7 Md€ pour la CVAE et 9,1 Md€ pour la TFPB. Sur l'augmentation en sept ans de $12 \mathrm{Md}$ ¿
${ }^{1}$ Montant tiré d'un rapport de I'Institut Montaigne et du Mouvement des Entreprises de Taille Intermédiaire (METI) d'octobre 2019 intitulé « Taxes sur la production - Préservons les entreprises dans les territoires"

${ }^{2}$ Ces deux montants perçus en 2018 au titre de la CFE et de la CVAE sont extraits du rapport 2019 de l'Observatoire des Finances et de la Gestion publique locale. 
du produit de ces trois principaux impôts prélevés au titre de la fiscalité directe locale, l'augmentation de 3,1 Md€ du produit de la TFPB en a représenté plus du quart, ce qui devrait appeler, pour l'avenir, une analyse et une vigilance particulières sur son évolution.

\section{Le risque de surimposition des entreprises au titre de la fiscalité directe locale peut menacer leur compétitivité et leur pérennité, notamment dans les territoires les plus fragiles}

\section{A. Une fiscalité relativement lourde pesant sur les coûts de production des entreprises}

Au sein de l'Union européenne, la France est le deuxième pays après la Suède dont la fiscalité pesant sur les coûts de production des entreprises est la plus importante en pourcentage du total de la fiscalité qui leur est imposée. En 2016, en effet, $25 \%$ de ce total revenait à la fiscalité imposée sur la production, contre $2 \%$ en Allemagne, principal pays concurrent en Europe.

La répartition de la fiscalité française sur les entreprises était la suivante:

- Impôts sur la production : 25 \%

- Impôts sur la consommation (notamment la TVA) : $61 \%$

- Impôts directs hors impôts sur la production (notamment l'impôt sur les bénéfices) : $14 \%$.

Au sein de la fiscalité sur la production, la fiscalité prélevée par les collectivités locales en représentait $51 \%$, dont $24 \%$ pour la cotisation économique territoriale (CFE + CVAE), $10 \%$ pour le versement transport et $17 \%$ pour la TFPB 3 .

Si les impôts sur la consommation sont les plus lourds, ils pèsent sur le coût de tous les produits et services consommés en France, à la fois ceux qui sont produits en France et ceux qui sont importés, alors que les impôts sur la production ne pèsent que sur le coût des produits et services produits en France, ce qui désavantage particulièrement à cet égard la compétitivité des entreprises françaises, d'autant plus fortement qu'en France les impôts sur la production sont relativement plus élevés que chez leurs concurrents, du fait notamment de la fiscalité locale qui en représente plus de la moitié.

\section{B. Une fiscalité directe locale sur la production qui pénalise particulière- ment les entreprises installées hors du champ des métropoles, dans les territoires ruraux ou semi ou péri-ur- bains}

Les entreprises situées dans les territoires ruraux, semi ou péri-urbains, sont principalement des en- treprises industrielles, ou des entreprises de taille intermédiaire $(E T I)$, des $P M E$, ou des très petites entreprises (TPE) de nature artisanale, dont les facteurs de production comprennent, pour une large part, des bâtiments et des équipements formant l'assiette d'imposition à la TFPB et à la CFE.

En effet, en application de l'article 1499 du code général des impôts : "La valeur locative des immobilisations industrielles passibles de la taxe foncière sur les propriétés bâties est déterminée au regard du prix de revient des différents éléments qui composent ces propriétés... Elle est établie essentiellement sur des données comptables, reposant principalement sur le prix de revient des sols, terrains, constructions, matériels et outillage ».

Et selon l'article 1498 du code général des impôts applicable aux locaux professionnels autres que les locaux industriels: "La valeur locative de chaque propriété bâtie ou fraction de propriété bâtie est déterminée en fonction de l'état du marché locatif à la date de référence du 1er janvier 2013, sous réserve de la mise à jour qui doit intervenir l'année qui suit le renouvellement général des conseils municipaux.

Elle est obtenue selon des tarifs par mètre carré déterminés sur la base des loyers moyens constatés dans chaque secteur d'évaluation par catégorie de propriétés et appliqués à la surface pondérée des locaux obtenue à partir de la superficie de leurs différentes parties, réduite, le cas échéant, au moyen de coefficients fixés par décret, pour tenir compte de leur utilisation et de leurs caractéristiques physiques respectives».

Contrairement aux entreprises de services, particulièrement les plus grosses d'entre-elles, préférentiellement situées sur le territoire des métropoles et des grandes agglomérations, dont la fiscalité directe repose principalement sur le facteur travail, la fiscalité directe locale des ETI, PME et TPE, plutôt situées dans les territoires ruraux, semi ou péri-urbains, repose, pour une large part, sur la valeur de leurs bâtiments et de leurs équipements qui forment l'assiette de l'imposition à la TFPB et à la CFE. sous-partie II.A sont extraites

du rapport précité de l'Institut Montaigne et du METI. 
D'où le risque de surimposition, dans ces territoires, qui apparaît particulièrement marqué pour la TFPB dans un contexte de disparition progressive de la majeure partie de la TH et de transfert aux communes de la part départementale de la TFPB. Dans ces dernières, en effet, pourrait apparaître une tentation de repli sur la seule ressource de TFPB, en substitution de la ressource de TH dont la compensation par L'État ne pourra qu'être nécessairement contenue dans la limite d'un certain plafond et selon des critères qui dépendront des propres marges de manœuvre budgétaires de l'État.

Ce risque de surimposition peut être contreproductif par un assèchement de la matière imposable dans les territoires communaux les plus fragiles, en conduisant les entreprises à quitter ces territoires pour s'installer dans un pays étranger, ou à cesser leur activité, en raison d'une fiscalité locale qui alourdit à l'excès leurs coûts de production.

La suppression de la taxe professionnelle en 2010 et son remplacement par la cotisation économique territoriale aura permis aux entreprises d'économiser une charge fiscale de $10 \mathrm{Md}$. Mais depuis cette réforme, I'alourdissement de la charge relative de la TFPB au sein de la fiscalité directe locale a, en partie, réduit cette économie.
Les Pouvoirs publics semblent avoir commencé à prendre conscience du risque causé par cette évolution si l'on en croit les dernières mesures prévues par la loi de finances pour 2020 en faveur des TPE et des PME. II y est, en effet, prévu des exonérations de CET et de TFPB au profit des TPE dans les communes rurales isolées pour une période limitée couvrant les quatre années de 2020 à 2023, ainsi que des exonérations des mêmes impôts, pour la même période, en faveur des PME dans les communes ayant conclu une convention d'opération de revitalisation de territoire.

Ces mesures partielles ne devraient pas conduire à s'abstraire d'une réflexion plus large sur le poids de la fiscalité directe locale pesant sur les coûts de production des entreprises implantées dans les territoires français les plus fragiles, notamment à l'aube d'une période où apparaît un risque de surimposition au titre de la TFPB pesant sur les entreprises du fait des incertitudes qui, à raison, se font jour quant aux conditions de compensation par l'État des ressources que les communes tiraient du prélèvement de la taxe d'habitation appelé à être supprimé pour la majeure partie de cette taxe. 